\title{
Pathophysiology of stress granules: An emerging link to diseases (Review)
}

\author{
JIHUI WANG, YIXIA GAN, JIAN CAO, XUEFEN DONG and WEI OUYANG \\ Department of Kinesiology and Human Sciences, College of Physical Education and Health Sciences, \\ Zhejiang Normal University, Jinhua, Zhejiang 321004, P.R. China
}

Received December 13, 2021; Accepted January 21, 2022

DOI: 10.3892/ijmm.2022.5099

\begin{abstract}
Under unfavorable environmental conditions, eukaryotic cells may form stress granules (SGs) in the cytosol to protect against injury and promote cell survival. The initiation, mRNA and protein composition, distribution and degradation of SGs are subject to multiple intracellular post-translational modifications and signaling pathways to cope with stress damage. Despite accumulated comprehensive knowledge of their composition and dynamics, the function of SGs remains poorly understood. When the stress persists, aberrant and/or persistent intracellular SGs and aggregation of SGs-related proteins may lead to various diseases. In the present article, the research progress regarding the generation, modification and function of SGs was reviewed. The regulatory effects and influencing factors of SGs in the development of tumors, cardiovascular diseases, viral infections and neurodegenerative diseases were also summarized, which may provide novel insight for preventing and treating SG-related diseases.
\end{abstract}

\section{Contents}

1. Introduction

2. Formation of SGs and influencing factors

3. Functions of SGs

4. SGs involved in cell senescence and tumors

5. SGs involved in cardiovascular disease

6. SGs involved in viral infection

7. SGs involved in neurodegenerative diseases

8. Conclusions

Correspondence to: Dr Wei Ouyang, Department of Kinesiology and Human Sciences, College of Physical Education and Health Sciences, Zhejiang Normal University, 28th Building, 688 Yinbing Road, Jinhua, Zhejiang 321004, P.R. China

E-mail: wouyang@zjnu.cn

Key words: stress granule, mRNA, RNA binding protein, liquid-liquid phase separation, assembly, diseases

\section{Introduction}

When facing the dangers of oxidative stress, temperature changes, toxins, hypoxia and osmotic pressure changes, eukaryotic cells produce a series of comprehensive stress responses in order to resist the above-mentioned unfavorable conditions and reduce damage (1). These include the production of membraneless messenger ribonucleoprotein (mRNP) particles, such as stress granules (SGs) and P bodies (PBs) in the cytoplasm (1-4). The aberrant assembly or disassembly of SGs has pathological implications in cancer (5-7) and viral infection $(8,9)$. In addition, SGs are associated with increased rates of aging-related conditions such as cardiovascular disease $(10,11)$ and neurodegenerative disorders (12-15). Therefore, a better understanding of the initiation, assembly and pathological assembly of SGs in these diseases may help to discover different target molecules and help to develop precise pharmacological interventions. The present article provides a review of current findings on the formation, composition, dynamics, function and involvement of SGs in different diseases.

\section{Formation of SGs and influencing factors}

When faced with a stress stimulus, the four monitoring kinases including protein kinase R (PKR), PKR-like endoplasmic reticulum kinase (PERK), heme-regulated kinase inhibitor and general control nonderepressible 2 (GCN2) in eukaryotic cells are activated and the Ser51 site of the translation initiation factor eukaryotic translation initiation factor 2A (eIF2) is phosphorylated. As a result, the eIF2-GTP-tRNAiMet ternary complex required for normal translation initiation is gradually depleted and the ribosomes disintegrate and stagnant $48 \mathrm{~S}$ preinitiation complexes (PICs), PICs including translation initiation factor, 40S ribosomal subunit, untranslated mRNA and RNA binding protein are generated, which aggregate to form the 'nucleus' of SGs and further combine with RNA-binding proteins (RBPs) to form the 'shell' of SGs (16-18). Furthermore, the activated 'shell' may exchange its components with ribosomes, PBs and other structures in the cytoplasm $(4,18-20)$, thus regulating the transport, translation, isolation and degradation of RNA $(2,3)$. The low-complexity domains (LCDs) contained in RBPs are critical to the formation of the 'core' and 'shell' of SGs. This region may mediate specific protein-protein, protein-RNA 
and/or RNA-RNA cross-linking and aggregation through liquid-liquid phase separation (LLPS), thereby condensing into SGs particles in the cytoplasm $(2,3)$. In addition, the formation and depolymerization of SGs are an energy-consuming process and multiple ATP-dependent complexes affect the assembly of SGs. For instance, the Chaperonin-Containing T complex inhibits the assembly of SGs, while the mini-chromosome maintenance and RuvB-like helicase complexes promote the survival of SGs $(20,21)$.

RBPs are regulated by a variety of intracellular signaling pathways, thereby affecting translation/protein synthesis. Under unfavorable circumstances, intracellular DNA-binding proteins and RBPs join SGs, such as TAR DNA-binding protein 43 (TDP-43), fused in sarcoma (FUS), GTPase-activating protein $\mathrm{SH} 3$ domain-binding protein (G3BP) SG assembly factor $1 / 2$ (G3BP1/2), ubiquitin specific peptidase 10 (USP10), cell cycle associated protein 1 (Caprin1), T-cell intracytoplasmic antigen 1 (TIA1) cytotoxic granule associated RNA binding protein (TIA1)/TIA1-related protein (TIAR) and heat shock proteins (Hsp)40/70 (18,21-24), participating in different stresses and the pathological process of disease (Table I). G3BP1/2, Caprin1, USP10 and TIA1/R have important roles in the formation of SGs (23). Among them, G3BP1/2 is regulated by the phosphorylation site of S149 and may bind to Caprin1 or USP10; binding to Caprin1 may promote the formation of SGs, while binding to USP10 may inhibit the formation of SGs (23). G3BP cross-links with $40 \mathrm{~S}$ ribosomal subunits through its arginine- and glycin-rich motif RGG, which is also one of the necessary conditions for G3BP to mediate the formation of SGs (23). In addition, TIA-1 or TIAR may also promote the formation of SGs alone $(23,25)$.

According to the translation initiation factors and RBPs contained, SGs may be divided into different subtypes. For instance, type I SGs are classical SGs. The formation process includes phosphorylation of eIF2 $\alpha$, which requires G3BP and 48S PICs. Its components include eIF3, but lack eIF2 and eIF5. The formation of type II SGs includes eIF4A inactivation and still requires G3BP. The group of type II SGs includes eIF2, eIF3 and eIF5. However, type III SGs lack eIF3 $(18,26,27)$, display reduced recruitment of $40 \mathrm{~S}$ ribosomal subunit (26) and are less dynamic (28). The formation of type III SGs is independent of the phosphorylation of eIF $2 \alpha$ and may be triggered by sodium selenite (26), glucose starvation (27) and nitric oxide (NO) (28). The different functions and properties of the diverse SG subtypes remain to be fully elucidated, but different SG subtypes may exert opposite effects on cellular metabolism and survival.

There is a full interaction between mRNA and RBPs in SGs. RBPs may act on mRNA to regulate its metabolism and function; conversely, mRNA may also be combined with RBPs to affect the subsequent functions of RBPs (2). After the separation between SGs, fusion may also form new SGs (29). The formation and depolymerization of SGs are also affected by a variety of post-translational modification factors. For instance, the phosphorylation of eIF $2 \alpha$ at Ser51 regulates the initiation of SGs; phosphorylation of tristetraprolin may regulate the interaction between SGs and PBs; and phosphorylation of growth factor receptor bound protein 7 may promote heat shock-induced depolymerization of SGs (18). The demethylation of G3BP1 and ubiquitin-associated protein 2 like (UBAP2L) protein arginine may promote the production of SGs $(30,31)$. The UBAP2L RGG motif is able to mediate the enrollment of SG components, including mRNPs, RBPs and ribosomal subunits $(30,31)$. The protein arginine methyltransferase 1 asymmetrically dimethylated UBAP2L by targeting the RGG motif, thus compromising SG assembly (30). Therefore, the RGG motif may serve as a critical interface for SG assembly. The RGG motif and arginine methylation/demethylation are involved in the regulation of the downstream nucleic acid binding, protein-protein interactions and signal transduction.

\section{Functions of SGs}

In eukaryotic cells, there is a competitive relationship between the signaling pathways that govern protein translation and mRNA degradation, which jointly regulate cell gene expression under different conditions. Both SGs and PBs are membraneless mRNP particles produced by LLPS from local high concentrations of RBPs and RNA in order to adapt to environmental changes $(24,29)$. Due to their non-membrane characteristics, SGs and PBs may serve as a stress signal processing center where various signaling pathways converge and may exchange components. Therefore, SGs and PBs share a variety of RBPs and RNA components (4).

When faced with external, unfavorable factors, cells suspend most of their protein synthesis to conserve energy. The mRNAs encoding housekeeping proteins (e.g., $\beta$-actin, $\beta$-tubulin and GAPDH) in the ribosome are transported to SGs for temporary storage $(24,32)$. The mRNAs encoding other proteins are transferred to PBs or other mRNPs for further storage or degradation $(18,24,29,32)$. However, certain transcripts involved in the stress response of SGs are allowed to be translated to maintain cell survival. For instance, activating transcription factor 4 and HSP70 help reduce misfolding during protein expression $(32,33)$. In addition, the pro-apoptotic protein receptor for activated $\mathrm{C}$ kinase 1 (RACK1) is 'isolated' in SGs, so that the MAP three kinase 1 (MTK1)-stress activated protein kinase (SAPK) signaling pathway that depends on RACK1 is blocked (34) and the activation of caspase-3 is reduced (35), thereby inhibiting the apoptotic signaling cascade response to promote cell survival.

When oxidative stress, heat shock and hypoxia, and other harmful stimuli persist, chronic stress may occur. Continuous cell stress response may induce mutations in RBPs, block autophagy pathways and cause deposition of disease-related proteins, leading to the breakdown of the stability/degradation balance of SGs, continuous accumulation of SGs, as well as degeneration and deposition and/or formation of abnormal SGs. Therefore, SGs are a key factor that determines the fate of mRNA when cells face stress and affect the translation, stabilization, transport and degradation of mRNA (Fig. 1) (18,36,37). When the subsequent stress crisis is relieved, eIF $2 \alpha$ is dephosphorylated, which may restore the normal translation initiation function and the normal protein translation process of the cell is restored $(21,36)$. Therefore, the main function of SGs is to help cells reorganize the translation process in protein synthesis, store housekeeping protein mRNA and solve the survival crisis first when faced with unfavorable environments. However, the persistence of SGs and the imbalance of SG 
Table I. Relations between specific stress granule initiation factors, proteins and different types of disease.

\begin{tabular}{|c|c|c|c|c|c|c|}
\hline \multirow{2}{*}{$\begin{array}{l}\text { Initiation factors } \\
\text { and RBPs }\end{array}$} & \multirow[b]{2}{*}{ Treatment/effectors } & \multicolumn{5}{|c|}{ Disease, Refs. for established relations } \\
\hline & & Tumor & VI & $\mathrm{AD}$ & ALS/FTD & $\mathrm{PD}$ \\
\hline $\mathrm{eIF} 2 \alpha$ & $\begin{array}{l}\text { Bortezomib, cisplatin, } \\
\text { etoposide, morusin, } \\
\text { NO, IL-19, PRV, EV71, } \\
\text { MERS-CoV, EBOV, } \\
\text { GCN2, PKR, PERK, } \\
\text { TDP-43 }\end{array}$ & $(27,34,41,42)$ & $(52,56,57,65)$ & $(74-76,102)$ & $(74,76)$ & \\
\hline eIF3, eIF4 & $\begin{array}{l}\text { NO, EV71, EBOV, PKR, } \\
\text { PERK, rapamycin, } \\
\text { DJ-1, COI }\end{array}$ & $(27)$ & $(56,57)$ & (74) & $(74,103)$ & $(100)$ \\
\hline $\begin{array}{l}\text { G3BP1 or } \\
\text { G3BP2 }\end{array}$ & $\begin{array}{l}\text { Cisplatin, etoposide, } \\
\text { morusin, NO, SASP, } \\
\text { SART3, YB-1, PKM2, } \\
\text { IL-19, LRP6, RIG-I } \\
\text { C108, CrPV-1A, PV, } \\
\text { EBOV, SOD1, COI }\end{array}$ & $\begin{array}{c}(27,34,35,37 \\
41,46,47)\end{array}$ & $(53,55,57)$ & $(84,103)$ & $(84,103)$ & \\
\hline $\begin{array}{l}\text { G3BP1 and } \\
\text { G3BP2 }\end{array}$ & $\begin{array}{l}\text { Bortezomib, MERS-CoV, } \\
\text { IFITM1, FMDV }\end{array}$ & $(42,45)$ & $(54,65)$ & & & \\
\hline RACK1 & NO, morusin, 5-Fu, DJ-1 & $(25,27,34)$ & & & & $(101)$ \\
\hline TIA1 & $\begin{array}{l}\text { NO, EV71, MERS-CoV, } \\
\text { CrPV-1A, YB-1, Tau, } \\
\text { DJ-1, COI }\end{array}$ & $(27)$ & $(53,56,65)$ & $(35,82-84)$ & $(35,82-84,103)$ & $(100)$ \\
\hline TDP-43 & $\begin{array}{l}\text { PKR, PERK, RRM, } \\
\text { SINE, tau, COI }\end{array}$ & & & $(90)$ & $(76,78,79,103)$ & \\
\hline FUS & Hpo, JNK, AchR, COI & & & & $(86,87,103)$ & \\
\hline hnRNPs & PrLD, DJ-1, MAP 1B-LC1 & $(104)$ & & & $(88,89)$ & $(100)$ \\
\hline \multicolumn{7}{|c|}{$\begin{array}{l}\text { CVD, cardiovascular diseases; VI, viral infections; AD, Alzheimer's disease; ALS, amyotrophic lateral sclerosis; FTD, frontotemporal } \\
\text { dementia; PD, Parkinson's disease; AchR, acetylcholine receptor; C108, G3BP antibody; COI, chronic optogenetic induction; CrPV-1A, cricket } \\
\text { paralysis virus 1A protein; DJ-1, protein deglycase; EBOV, ebola virus; eIF2, eukaryotic translation initiation factor 2; EV71, enterovirus 71; } \\
\text { FMDV, foot-and-mouth disease viruses; FUS, fused in sarcoma; G3BP1, GTPase-activating protein SH3 domain-binding protein 1; G3BP2, } \\
\text { GTPase-activating protein SH3 domain-binding protein2; GCN2, general control nonderepressible 2; hpo, protein kinase Hippo; IFITM, } \\
\text { interferon inducible transmembrane proteins; JNK, cJun-N-terminal kinase; LRP6, low-density lipoprotein receptor-related protein 6; } \\
\text { MAP 1B-LC1, microtubule-associated protein 1B light chain; NO, nitric oxide; PERK, protein kinase RNA-like endoplasmic reticulum } \\
\text { kinase; PKM2, pyruvate kinase M2; PKR, protein kinase R; PrLD, prion-like domain; PV, poliovirus; PRV, pseudorabies virus; RACK1, } \\
\text { receptor for activated C kinase 1; RBP, RNA-binding protein; RIG-I, retinoic acid-inducible gene I; RRM, RNA recognition motif; SART3, } \\
\text { spliceosome associated factor 3; SASP, senescent-associated secretory phenotype; SINE, selective inhibitor of nuclear export; SOD1, Cu/Zn } \\
\text { superoxide dismutase; TDP-43, TAR DNA-binding protein 43; TIA1, T-cell intracytoplasmic antigen 1; YB-1, Y-box binding protein 1; 5-Fu, } \\
\text { 5-fluorouracil. }\end{array}$} \\
\hline
\end{tabular}

production/decomposition are also the main reasons for the degeneration and hydrolysis of important cellular mRNAs and may promote the occurrence of numerous diseases, including cancer, cardiovascular diseases, viral infections and neurodegenerative diseases $(18,21,36)$.

\section{SGs involved in cell senescence and tumors}

Cell senescence is one of the main pathogenic factors of cancer. During ageing, cells in the human body gradually become senescent. Under chronic oxidative stress, senescent cells inhibit the production of SGs in the cytoplasm, which may be accompanied by heat shock response and autophagy, which further promote the decomposition of SGs (38). The key factors, HSP70 and autophagy related 5, which inhibit the heat-shock response, as well as the autophagy pathway, may restore the ability of senescent cells to generate SGs during chronic oxidative stress (38). Aging-related secreted phenotype (SASP) factors are closely related to the occurrence of tumors $(39,40)$. A recent study suggested that G3BP1 is not only the key RBP for cell stress response and $\mathrm{SG}$ generation, but also a necessary factor for activating SASP factors. G3BP1 may activate the NF- $\kappa$ B and signal transducer and activator of transcription 3 signaling pathways through cyclic GMP-AMP synthase (cGAS) to promote the expression of SASP protein; depleting G3BP1 or inhibiting the cGAS pathway may prevent the expression of SASP factors and reduce the occurrence and migration of tumors without affecting the normal senescence of cells (39). 


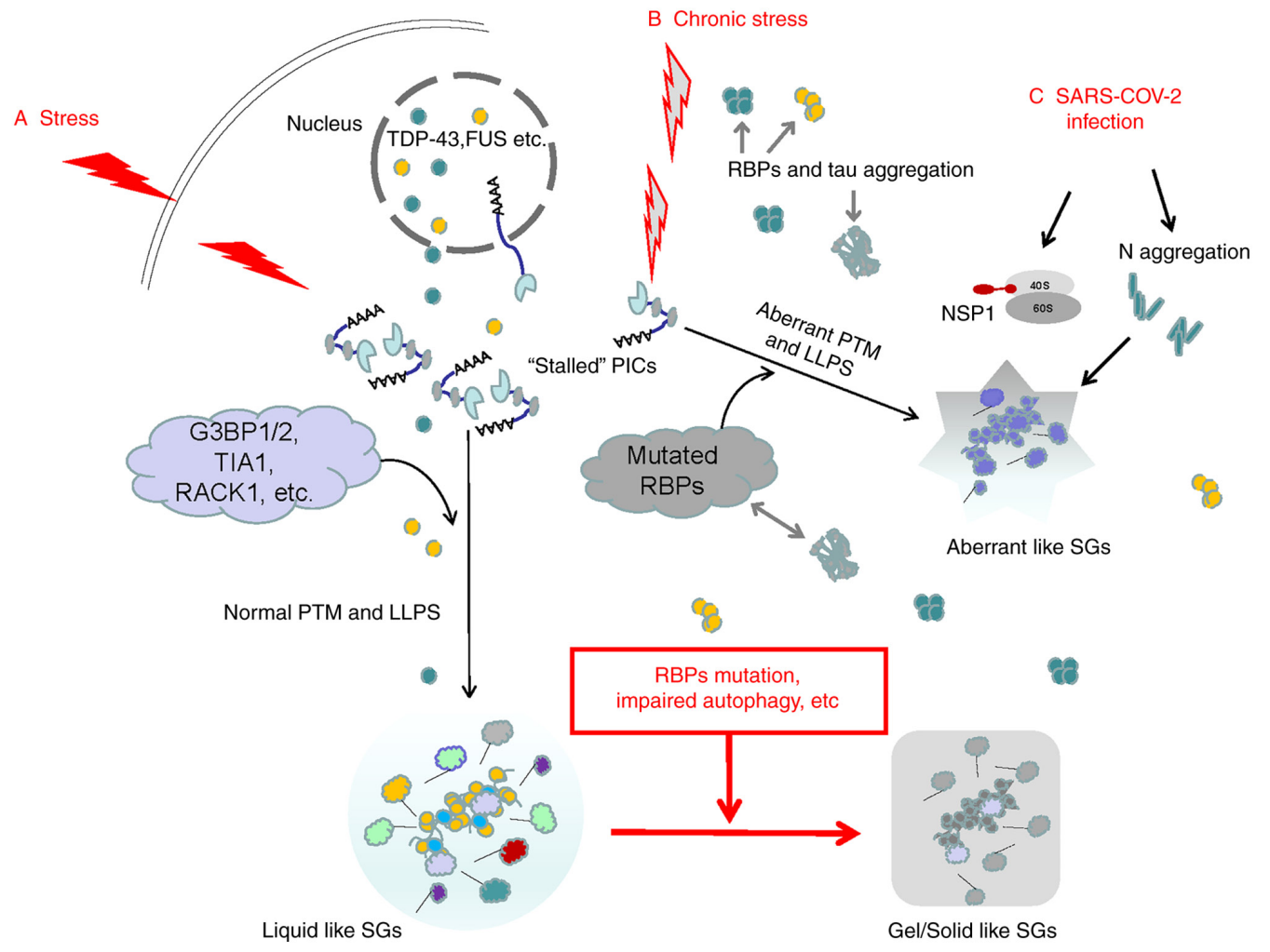

Figure 1. Possible mechanisms of acute and chronic stress-inducing different types of SGs. (A) When subjected to acute stresses, the stalled 48S PICs in eukaryotic cells serve as the 'nucleus' of SGs. The SG 'shell' consists of various dynamic RBPs. The assembly of SG 'cores' and 'shells' is largely driven by LLPS. The mRNA in SGs is able to resume translation after the stress is relieved. (B) Chronic stress may induce mutations and aggregation of DNA-binding proteins and RBPs, leading to abnormal LLPS and generating aberrant SGs. The deposited tau may cross-link with RBPs. As chronic stress persists, RBPs in normal SGs may also undergo mutations and the autophagy hydrolysis pathway of SGs is blocked. Normal SGs gradually change from liquid to gel/solid and persist, eventually inducing various diseases. (C) The typical RBPs in SGs, such as G3BP1, are the main targets of various viruses. The N protein in SARS-COV-2 integrates with G3BP1 into SGs, promoting the degeneration of SGs. In addition, after SARS-COV-2 enters human host cells, the initially synthesized NSP1 may block the channel of host mRNA to enter the 40S subunit but promote viral replication. SG, stress granule; RBP, RNA-binding protein; SARS-COV-2, severe acute respiratory syndrome-coronavirus disease; LLPS, liquid-liquid phase separation; G3BP1, G3BP SG assembly factor 1; TIA1, TIA1 cytotoxic granule associated RNA binding protein; RACK1, receptor for activated C kinase 1; NSP1, non-structural protein 1; PICs, preinitiation complexes; PTM, post-translational modification.

The survival of tumor cells faces numerous unfavorable conditions, such as hypoxia, nutrient deficiency, reactive oxygen species and high osmotic pressure. Tumor cells may pursue the path of generating SGs to selectively perform mRNA translation to regulate cell signal transduction pathways, metabolism and stress response to promote their own survival $(1,41,42)$. For instance, in liver cancer cells, PI3K and MAPK/p38 activate the mechanistic target of rapamycin (mTOR) complex 1-S6 kinase signaling pathway and phosphorylate eIF $2 \alpha$, eIF3, eIF4B and eIF5 to generate SGs (5-7), which may promote the development of liver cancer (6). Chemotherapy drugs commonly used in cancer treatment, such as bortezomib, cisplatin or etoposide, may stimulate tumor cells to generate SGs that depend on eIF2 $\alpha$ phosphorylation and resist the effects of drugs $(43,44)$. In tumor cells cultured in vitro, the plant-derived anti-cancer drug Sanxin may activate PKR and eIF2 $\alpha$ phosphorylation in cancer cells and induce the production of SGs (35). SGs may also regulate tumor-related signaling pathways and participate in tumor migration. By inhibiting the phosphorylation of eIF2 $\alpha$ and interfering with the recruitment of SGs or microtubule polymerization, tumor occurrence and metastasis may be inhibited $(43,45)$.

$\mathrm{NO}$ is one of the key factors in tumorigenesis, development or suppression, depending on the source and concentration of
NO. The high concentration of NO synthesized by inducible NO synthase in activated macrophages/lymphocytes may induce cell cycle arrest and apoptosis and exert anti-tumor effects. The low and intermediate concentrations of NO synthesized by tumors and endothelial cells interfere with normal cell DNA repair and promote the accumulation of mutations, tumor heterogeneity, angiogenesis, epithelial mesenchyme and tumor invasion (46). The different effects of NO in inhibiting or promoting tumorigenesis may be related to the regulation of different translation initiation factors by $\mathrm{NO}$, inducing the production of different subtypes of SGs in cancer cells and promoting the conversion between different subtypes of SGs. For instance, the lack of eIF3 in type II SGs may promote tumorigenesis (28).

Various RBPs also have a vital role in the generation of SGs in tumor cells and the promotion of tumor development (Table I). The anti-metabolite drug 5-fluorouracil (5-Fu) is widely used to treat solid tumors. However, tumors generally develop resistance to $5-\mathrm{Fu}$ in the later stages of treatment. Kaehler et al (25) indicated that during the process of 5-Fu inducing SGs in cancer cells, RACK1 was isolated in SGs, which inhibited MTK1-SAPK and downstream tumor apoptosis signaling pathways, resulting in a decrease in the effect of 5-Fu chemotherapy. Similarly, when Sanxin was used to treat tumors, RACK1 was isolated in SGs, which reduced 
the activation of caspase- 3 and the therapeutic effect of Sanxin (35). The results of in vitro experiments indicate that the use of comprehensive therapies such as hypoxia, arsenite and X-ray therapy may also induce the production of SGs containing RACK 1 in cancer cells, thereby reducing cancer cell apoptosis and resisting therapeutic intervention (34).

Compared with the control group, G3BP1 gene knockout in tumor cells significantly enhanced the sensitivity to Sanxin treatment (35). G3BP1/2 is able to regulate the translation of interferon-stimulated genes to promote tumor development and metastasis (47); G3BP2 was also reported to promote breast tumors by binding to squamous-cell carcinoma antigens (48). Studies on SGs of human sarcoma cells indicated that G3BP1 interacts with Y-box binding protein (YB-1) to jointly promote the production of SGs; at the same time, the increased expression of G3BP1 and YB-1 predicts a poor survival prognosis for tumor patients (49). In the SGs of tumor cells induced by sodium arsenite, the distributions of G3BP1 and TIA1/R are highly overlapped and may jointly regulate the stability and degradation of mRNA in SGs under stress conditions (37). Therefore, by regulating the phosphorylation of translation initiation factors, regulating the SGs-promoting effects of various RBPs, such as RACK1, G3BP1/2 and the downstream signaling pathways of SGs/RBPs, may reduce the incidence of tumors, improve the efficacy of tumor treatment drugs and reduce the mortality of patients with cancer.

\section{SGs involved in cardiovascular disease}

A recent study indicated that for monocytes/macrophages isolated from the serum of patients with myocardial infarction (MI) and coronary artery disease, hyperglycemia stimulation activated the pyruvate kinase isoenzyme type M2 (PKM2), followed by SGs marker protein G3BP1 production, intravascular plaque loosening and shedding; eventually, the risk of thrombosis was increased (10). A study on atherosclerotic plaques in low-density lipoprotein (LDL) receptor knockout mice suggested that increased expression of G3BP1 and poly-A binding protein (PABP) in macrophages and smooth muscles was significantly associated with the progression of atherosclerosis (11). In human coronary artery smooth muscle and macrophages cultured in vitro, application of oxidized LDL and mitochondrial/oxidative stress mediator may rapidly induce the production of SGs containing PABP and G3BP; while application of IL-19 may inhibit eIF2 $\alpha$ phosphorylation, PABP expression and SG production (11).

A molecular genetic study in humans and rodents indicated that the Wnt and LDL receptor-related protein (LRP) signaling pathway may be a key step in cardiometabolic diseases. For cultured mouse aortic smooth muscle cells, under the stimulation of glycerol phosphate, the expression of G3BP1, a marker protein of SGs in smooth muscle cells, increased through its C-terminal arginine methylation domain and mitochondrial antiviral signal protein retinoic acid-inducible gene I cross-linking to promote Wnt signaling related to arteriosclerosis and accelerated arteriosclerosis (50). The application of G3BP antagonist $\mathrm{C} 108$ may reduce G3BP1 methylation and Wnt signaling (50).

The mutation of human RNA binding motif protein 20 (RBM20) caused by gene mutation is closely related to congenital dilated cardiomyopathy. A clinical study indicated that mRNP particles containing RBM20 variants accumulate abnormally in the sarcoplasm of patients' myocardial cells and promote the fusion of biomolecules and SGs in the sarcoplasm (51). Therefore, the disorder of the protein components of mRNP particles, including SGs, is closely related to cardiovascular diseases such as MI and coronary artery disease, atherosclerosis and dilated cardiomyopathy. In addition, the above-mentioned diseases are mostly chronic diseases except for MI, which may cause intracellular protein translation, RBP dysregulation and the persistence of SGs, thereby promoting the development of cardiovascular diseases. For instance, acute coronary syndrome, otherwise known as type $1 \mathrm{MI}$, occurs as a result of vulnerable plaque rupture with following thrombus formation and arterial spasm and thus coronary occlusion (52). However, the activation of PKM2 may mediate NLR family pyrin domain containing 3 inflammasome and G3BP1 production, ultimately increasing plaque vulnerability/rupture and is associated with acute MI (10). Circular RNAs are a subclass of non-coding RNAs detected within mammalian cells and are generated from numerous protein-coding genes. It was recently reported that a circRNA transcript, circFndc $3 \mathrm{~b}$, is significantly downregulated in the post-MI mouse and human hearts. Overexpression of circFndc $3 b$ significantly enhanced vascular endothelial growth factor A expression via reducing the levels of SGs-related protein FUS (53). These results indicated that modulation of circRNA expression and/or SGs-related protein levels may represent a potential strategy to promote cardiac function and remodeling after MI. In the meantime, meticulous research is also required to determine the roles of SGs and RBPs in acute cardiovascular diseases such as MI.

\section{SGs involved in viral infection}

SGs produced by host cells have an important role in antiviral innate immunity (54). During virus attack, viral double-stranded RNA may activate PKR and PERK in host cells, phosphorylate eIF2 $\alpha$, generate SGs and inhibit the translation and replication of viral RNA; therefore, SGs have also become the target of numerous viral attacks $(8,9)$. Different viruses may affect the various stages of SGs, such as inhibiting the production of SGs or interfering with the components of SGs and the function of SGs $(8,55)$. Pseudorabies virus (PRV) may infect other mammals such as pigs, cattle, sheep, dogs and cats. The results of in vitro experiments suggest that PRV may inhibit the process of eIF $2 \alpha$ phosphorylation induced by sodium arsenite, inhibit the production of SGs and promote viral replication (56). The cricket paralysis virus (CrPV)-associated protein $\mathrm{CrPV}-1 \mathrm{~A}$ may induce the aggregation of G3BP1 and inhibit the production of SGs (57). Foot-and-mouth disease virus and equine rhinitis $A$ virus also inhibit the production of SGs by host cells by lysing G3BP1/2 (58).

Poliomyelitis virus (PV) may induce host cells to produce SGs at the initial stage of infection, but as time goes by, PV promotes the cleavage of G3BP1 through protease $3 \mathrm{C}$ and inhibits the production of SGs (59). Protease 2A in picornavirus, EV71, isolates host mRNA by cleaving eIF4GI to form atypical $\mathrm{SGs}$, but releases viral mRNA to promote virus replication (60). Ebola virus (EBOV) replicates in host cells 
and infection does not induce the production of SGs, and may inhibit the process of arsenite-induced SGs; EBOV virus particles may also inhibit the production of SGs induced by a variety of drug stresses (61). The effect of EBOV in inhibiting SGs may lie in the cross-linking between its C-terminal domain and G3BP1, eIF3 and eEF2 in SGs (61).

Coronavirus disease 2019, which is currently circulating globally, is a highly contagious disease caused by severe acute respiratory syndrome coronavirus 2 (SARS-COV-2) infection. The latest research has indicated that the SARS-COV-2 nucleocapsid $(\mathrm{N})$ protein is cross-linked with G3BP1 in the host cell SGs through LLPS to isolate G3BP1, thereby inhibiting the formation of SGs or promoting the degeneration of SGs, and is beneficial to the virus' self replication (62-64). Prasad et al (65) even observed that 116 human SG proteins directly interacted with SARS-CoV-2 proteins and they are involved in 430 different brain disorders.

SARS-COV-2 may also interfere with the initiation of SGs in the host cell by inhibiting translation initiation; for instance, after SARS-COV-2 enters the host cell, it first synthesizes non-structural protein 1 (NSP1), and NSP1 uses its $\mathrm{C}$-end to bind to the 40S subunit in the host cell ribosome complex, blocking the passage of host mRNA into the $40 \mathrm{~S}$ subunit; NSP1 may also bind to 43S PIC (66-68). In addition, SARS-COV-2 may also inhibit host cell translation and immune response through NSP1, but does not restrict the translation of the virus' own protein (66-68). In the host cell, the aggregated $\mathrm{N}$ protein and SARS-COV-2 RNA polymerase form a high-density complex through LLPS, which may effectively promote the translation of viral RNA and viral replication (62). MERS-CoV, similar to SARS-COV-2, inhibits PKR-mediated phosphorylation of eIF2 $\alpha$, prevents host cells from producing SGs and promotes viral mRNA translation (69). At present, as is common for viruses, new coronavirus variants keep on emerging, and certain strains may be more contagious and harmful. Whether the mutated SARS-COV-2 has other ways to inhibit host cell translation and immune response, inhibit the production of SGs in host cells and promote its own protein translation and virus replication requires further research.

The diversity of the above-mentioned viruses' attack methods in host cells highlights the importance of SGs in anti-viral defense. It is necessary to intervene with specific proteins according to the interaction between different viruses and the host cell's SG production process, and determine a treatment plan. For instance, for SARS-COV-2, it interferes with the LLPS process in the host cell, inhibits the accumulation of $\mathrm{N}$ in large quantities and interferes with the binding of NSP1 to the 40S subunit of the host cell's ribosome. However, a latest heavily mutated Omicron variant (B.1.1.529) puts scientists on alert due to its increased infectivity and ability to evade infection-blocking antibodies compared to the Delta and Alpha variants (70). In addition, certain key questions about this variant remain unanswered, such as whether Omicron is more transmissible and causes more severe or milder pathologies than other variants. A question related to the present review, namely how SGs/RBPs directly and indirectly regulate various signaling pathways to contribute to Omicron evading the antiviral immune response is also worthy of in-depth study.

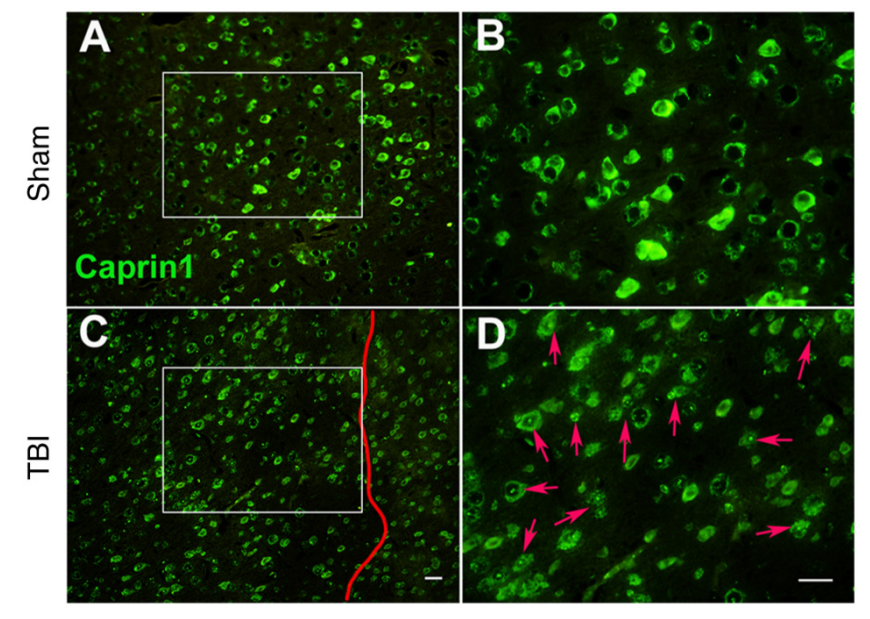

Figure 2. Moderate TBI induces the alteration of caprin1 expression in injured neurons of the C577BL/6J mouse motor cortex. (A and B) Sham group at magnifications of (A) x100 and (B) x200. (C and D) TBI group at (C) $\times 100$ and (D) $\times 200$ magnification (scale bars, $30 \mu \mathrm{m}$ ). B and D provide magnified windows of A and C, respectively. In TBI mice, the right somatosensory cortex had been subjected to a moderate fluid percussion pulse from 2.5 to $2.6 \mathrm{~atm}$. On day 8 post-injury, immunofluorescence analysis indicated that neurons in the sham animals exhibited clear and even caprin1 expression in the motor cortex; while the expression of caprin1 (stress granule-related RNA-binding proteins, as indicated by red arrows in D) in typical lesioned neurons on the left of the vertical red line in TBI animals was largely diffusive and uneven with numerous dots. However, caprin1 expression in neurons outside the lesion zone (on the right side of the vertical red line) was less affected. The experimental protocol is provided in Appendix S1. TBI, traumatic brain injury.

\section{SGs involved in neurodegenerative diseases}

Accompanied by chronic encephalopathy caused by brain aging, ischemia, traumatic brain injury and other factors such as secondary neuroinflammation, the nervous system of the elderly is prone to chronic stress and in turn, the occurrence of chronic stress is closely related to neurodegenerative disease (19,71). Among the mRNP particles induced by stress, SGs are most closely related to neurological diseases $(19,71)$. The above-mentioned risk factors, such as brain aging, cerebral ischemia, brain injury and neuroinflammation, may induce the generation of SGs in neurons, regulate the expression of RBPs (72-75), such as Caprin1 (Fig. 2), and induce irreversible accumulation of key RBPs and tau $(19,76)$.

In SGs, RBPs and deposited tau may be cross-linked, hindering the translation of RNA. Eventually, RBP and/or tau deposits aggregate and transform into an irreversible state of accumulation (77). The results of in vitro studies have indicated that under pathological conditions, SGs transform from liquid to viscous/solid amyloid and cause tau deposition (12-15), which promotes neurodegenerative diseases (77). The phosphorylation of neuron eIF2 $\alpha$ and eIF4 is mainly caused by the activation of signaling pathways such as mTOR, GCN2, PKR and PERK; therefore, these signaling pathways may also become targets for the development of neuroprotective drugs (78-80). The disordered LCDs in RBPs may regulate the liquid-solid transition of mRNPs. Under pathological conditions, the LCDs of RBPs exhibit a highly ordered structure, which promotes the aggregation of RBPs and the unidirectional transformation of mRNPs from liquid to solid, 
and generates pathological SGs $(13,81)$. Full-length tau with internal disorder may also pass through LLPS under pathological conditions and aggregate (15). Numerous proteins involved in the composition of SGs, such as TDP-43 (82-85), TIA1 $(86,87)$, G3BP1/2 $(88,89)$, FUS $(90,91)$ and heterogeneous nuclear ribonucleoproteins (hnRNPs) $(92,93)$, are related to the pathogenesis of neurodegenerative diseases (Table I).

Amyotrophic lateral sclerosis (ALS) and frontotemporal dementia (FTD) have the same pathological and genetic characteristics, and TDP-43 and FUS are closely related to ASL/FTD (82-84,90,91). TDP-43 also accumulates in the brains of patients with Alzheimer's disease (AD) $(85,94)$. In the brains of patients with $\mathrm{AD}$, in addition to $\beta$-amyloid and tau protein, TDP-43 may also accumulate in the limbic system; AD patients with full-length TDP accumulation have more obvious FTD symptoms (85). The latest research suggested that FUS is able to regulate the gene expression of acetylcholine receptors at the neuromuscular junction and FUS mutations may directly lead to ALS (91). In addition, the accumulation of FUS mutations may activate the protein kinase Hippo and JNK signaling pathways, accelerating neuronal degeneration and apoptosis (90). RBPs, such as TDP-43 and FUS, regulate the distribution and metabolism of RNA in cells by binding to coding and non-coding RNA; these RBPs may interact and participate in the pathogenesis of ALS/FTD $(95,96)$. Clinical autopsy results suggested the presence of a large number of TDP-43 accumulations in neurons of TIA 1 mutation carriers; TIA1 mutations may hinder the decomposition of SGs and promote the accumulation of TDP-43 in non-dynamic SGs (96).

Under stress, neurons may work with their intracellular SGs to degrade a large amount of RNA through the autophagy pathway $(18,19)$. When the crisis is removed, SGs are also eliminated through autophagy and the normal translation process is restored $(18,19)$. However, under continuous stress, the chromosome 9 open reading frame 72 mutation causes the SG autophagy pathway to be blocked and promotes the accumulation of TDP-43 in the cytoplasm $(97,98)$, leading to ALS/FTD $(99,100)$. In addition, the methylation of FUS arginine at R 218 by protein arginine methyltransferase 5 is a prerequisite step for the formation of the above-mentioned complex and the maintenance of the SG clearance mechanism (99). Mutations in p62 may also reduce the ability of neurons to clear SGs, resulting in neurotoxicity (100). Therefore, regulating the production/disaggregation balance of SGs in stress by arginine methyltransferase and p62 kinase drugs may help reduce the occurrence of neurodegenerative diseases. In the pathological process of AD and ALS/FTD, RBPs and tau in SGs may also interact with each other (94). Transgenic animal (87) and in vitro experiments (86) indicated that TIA 1 is able to promote tau phosphorylation and misfolding, and they then enter pathological SGs together, affecting the normal metabolism of RNA; tau may also regulate the distribution of TIA1 (86). In addition to long-term potentiation (LTP), long-term depression (LTD) is also involved in regulating the synaptic plasticity of hippocampal neurons and they jointly affect the formation of different types of memory. The phosphorylation of eIF $2 \alpha$ may reduce LTP, increase mGluR-LTD and affect learning and memory $(101,102)$.
DJ-1 is a protein/nucleic acid desaccharase encoded by the gene PARK7 related to familial Parkinson's disease (PD). It has numerous functions, such as anti-oxidation, transcription regulation and regulation of protein degradation. The expression of DJ-1 helps delay the pathological process of neurodegenerative diseases such as PD and AD (103). During acute stress, DJ-1 is able to combine with SGs to jointly regulate RNA metabolism and shunt, and exert a neuroprotective effect $(104,105)$. However, under chronic stress conditions, DJ-1 mutation promotes the transformation of SGs into pathological SGs and promotes the occurrence of PD (104). Therefore, eIF $2 \alpha$ phosphorylation, liquid-solid phase transformation of SGs, incorrect intracellular distribution of RBPs, RNA metabolism disorders and interactions between RBPs and tau or DJ-1 may all be targets for the development of drugs for neurodegenerative diseases.

\section{Conclusions}

The production of SGs by eukaryotic cells is an important means of resisting various stress injuries and maintaining cell survival. SGs contain translation initiation factors, untranslated mRNA and RBPs. During the stress process, numerous signaling pathways/proteins affect the distribution, assembly and degradation of SGs. SGs may also change a variety of intracellular signaling pathways by isolating various messenger proteins. Therefore, SGs may be combined with other mRNPs, such as PBs, to become the 'stress signal processing center' of cells under stress conditions. However, there are still numerous unknowns about SGs. For instance, it remains elusive how various intracellular signaling pathways regulate the initiation factors to affect the initiation, nucleation and cross-linking process of SGs with RBPs. Similarly, how various RBPs inside and outside SGs regulate the storage, retranslation and degradation of specific mRNAs is also undetermined. In the acute phase of stress, the assembly/disassembly of SGs is in a dynamic equilibrium. As the stress continues, SGs may transform into aggregated pathological SGs. Possibly, varied signaling pathways engage in regulating the assembly/disassembly of SGs under acute or chronic stress conditions. The coding and non-coding mRNA components of SGs and the relationships between different RBP mutations and diseases are also worthy of in-depth study. Therefore, it is necessary to clarify the various components of SGs, including mRNA and RBPs, and the roadmap for the occurrence of various diseases, so as to provide a theoretical basis for the design and development of drugs for SG-related diseases.

\section{Acknowledgements}

The authors thank Miss Sophie Ouyang (third-year grade medical student of UPenn Nursing School, Bachelor of Science in Nursing Program, Philadelphia, USA) for language editing the article.

\section{Funding}

This work was supported by grants from the Natural Science Foundation of Zhejiang, China (grant no. LY20H150001), the National College Student Innovation and Entrepreneurship 
Training Program (grant no. 201710345005) and the College Student Innovation and Entrepreneurship Training Program of Zhejiang, China (grant no. 2019R404011).

\section{Availability of data and materials}

The datasets generated and/or used during the current study are available from the corresponding author on reasonable request.

\section{Authors' contributions}

All authors participated in the design and revision of the manuscript. JW, YG and WO reviewed the literature and shared the writing of several drafts of the manuscript. JC and $\mathrm{XD}$ provided the figures and table. WO performed the final review of the manuscript. All authors read and approved the final manuscript. Data authentication is not applicable.

\section{Ethics approval and consent to participate}

All procedures performed to produce Fig. 2 were performed with the approval of the Institutional Animal Care and Use Committee (Zhejiang Normal University, Jinhua, China) and in accordance with the laws and regulations. The Care and Use Standard for Laboratory Animals (China Ministry of Health publication) and the National Institute of Health Guide for the Care and Use of Laboratory Animals (2011 edition) were followed.

\section{Patient consent for publication}

Not applicable.

\section{Competing interests}

The authors declare that they have no competing interests.

\section{References}

1. Ivanov P, Kedersha N and Anderson P: Stress granules and processing bodies in translational control. Cold Spring Harb Perspect Biol 11: a032813, 2019.

2. Hentze MW, Castello A, Schwarzl T and Preiss T: A brave new world of RNA-binding proteins. Nat Rev Mol Cell Biol 19: 327-341, 2018.

3. Shin Y and Brangwynne CP: Liquid phase condensation in cell physiology and disease. Science 357: eaaf4382, 2017.

4. Riggs CL, Kedersha N, Ivanov P and Anderson P: Mammalian stress granules and P bodies at a glance. J Cell Sci 133: jcs242487, 2020.

5. Heberle AM, Razquin Navas P, Langelaar-Makkinje M, Kasack K, Sadik A, Faessler E, Hahn U, Marx-Stoelting P, Opitz CA, Sers C, et al: The PI3K and MAPK/p38 pathways control stress granule assembly in a hierarchical manner. Life Sci Alliance 2: e201800257, 2019.

6. Golob-Schwarzl N, Krassnig S, Toeglhofer AM, Park YN, Gogg-Kamerer M, Vierlinger K, Schröder F, Rhee H, Schicho R, Fickert $\mathrm{P}$ and Haybaeck J: New liver cancer biomarkers: $\mathrm{PI} 3 \mathrm{~K} / \mathrm{AKT} / \mathrm{mTOR}$ pathway members and eukaryotic translation initiation factors. Eur J Cancer 83: 56-70, 2017.

7. Sfakianos AP, Mellor LE, Pang YF, Kritsiligkou P, Needs H, Abou-Hamdan H, Désaubry L, Poulin GB, Ashe MP and Whitmarsh AJ: The mTOR-S6 kinase pathway promotes stress granule assembly. Cell Death Differ 25: 1766-1780, 2018.

8. Onomoto K, Yoneyama M, Fung G, Kato H and Fujita T: Antiviral innate immunity and stress granule responses. Trends Immunol 35: 420-428, 2014.
9. McCormick C and Khaperskyy DA: Translation inhibition and stress granules in the antiviral immune response. Nat Rev Immunol 17: 647-660, 2017.

10. Li Q, Leng K, Liu Y, Sun H, Gao J, Ren Q, Zhou T, Dong J and Xia J: The impact of hyperglycaemia on PKM2-mediated NLRP3 inflammasome/stress granule signalling in macrophages and its correlation with plaque vulnerability: An in vivo and in vitro study. Metabolism 107: 154231, 2020.

11. Herman AB, Silva Afonso M, Kelemen SE, Ray M, Vrakas CN, Burke AC, Scalia RG, Moore K and Autieri MV: Regulation of stress granule formation by inflammation, vascular injury, and atherosclerosis. Arterioscler Thromb Vasc Biol 39: 2014-2027, 2019.

12. Patel A, Lee HO, Jawerth L, Maharana S, Jahnel M, Hein MY, Stoynov S, Mahamid J, Saha S, Franzmann TM, et al: A Liquid-to-Solid phase transition of the ALS Protein FUS accelerated by disease mutation. Cell 162: 1066-1077, 2015.

13. Ramaswami M, Taylor JP and Parker R: Altered ribostasis: RNA-protein granules in degenerative disorders. Cell 154: 727-736, 2013.

14. Ambadipudi S, Biernat J, Riedel D, Mandelkow E and Zweckstetter M: Liquid-liquid phase separation of the microtubule-binding repeats of the Alzheimer-related protein Tau. Nat Commu 8: 275, 2017.

15. Wegmann S, Eftekharzadeh B, Tepper K, Zoltowska KM, Bennett RE, Dujardin S, Laskowski PR, MacKenzie D, Kamath T, Commins C, et al: Tau protein liquid-liquid phase separation can initiate tau aggregation. EMBO J 37: e98049, 2018.

16. Kedersha N, Chen S, Gilks N, Li W, Miller IJ, Stahl J and Anderson P: Evidence that ternary complex (eIF2-GTP-tRNA(i) (Met))-deficient preinitiation complexes are core constituents of mammalian stress granules. Mol Biol Cell 13: 195-210, 2002.

17. Anderson P and Kedersha N: Visibly stressed: The role of eIF2, TIA-1, and stress granules in protein translation. Cell Stress Chaperones 7: 213-221, 2002.

18. Hofmann S, Kedersha N, Anderson P and Ivanov P: Molecular mechanisms of stress granule assembly and disassembly. Biochim Biophys Acta Mol Cell Res 1868: 118876, 2021.

19. Wolozin B and Ivanov P: Stress granules and neurodegeneration. Na Rev Neurosci 20: 649-666, 2019.

20. Jain S, Wheeler JR, Walters RW, Agrawal A, Barsic A and Parker R: ATPase-modulated stress granules contain a diverse proteome and substructure. Cell 164: 487-498, 2016.

21. Protter DSW and Parker R: Principles and properties of stress granules. Trends Cell Biol 26: 668-679, 2016.

22. Markmiller S, Soltanieh S, Server KL, Mak R, Jin W, Fang MY, Luo EC, Krach F, Yang D, Sen A, et al: Context-dependent and disease-specific diversity in protein interactions within stress granules. Cell 172: 590-604.e13, 2018.

23. Kedersha N, Panas MD, Achorn CA, Lyons S, Tisdale S, Hickman T, Thomas M, Lieberman J, McInerney GM, Ivanov P and Anderson P: G3BP-Caprin1-USP10 complexes mediate stress granule condensation and associate with $40 \mathrm{~S}$ subunits. J Cell Biol 212: 845-860, 2016.

24. Anderson P and Kedersha N: Stress granules: The Tao of RNA triage. Trends Biochem Sci 33: 141-150, 2008.

25. Kaehler C, Isensee J, Hucho T, Lehrach $\mathrm{H}$ and Krobitsch S: 5-fluorouracil affects assembly of stress granules based on RNA incorporation. Nucleic Acids Res 42: 6436-6447, 2014.

26. Fujimura K, Sasaki AT and Anderson P: Selenite targets eIF4E-binding protein-1 to inhibit translation initiation and induce the assembly of non-canonical stress granules. Nucleic Acids Res 40: 8099-8110, 2012.

27. Ohn T, Kedersha N, Hickman T, Tisdale S and Anderson P: A functional RNAi screen links O-GlcNAc modification of ribosomal proteins to stress granule and processing body assembly. Nat Cell Biol 10: 1224-1231, 2008.

28. Aulas A, Lyons SM, Fay MM, Anderson P and Ivanov P: Nitric oxide triggers the assembly of 'type II' stress granules linked to decreased cell viability. Cell Death Dis 9: 1129, 2018.

29. Anderson P and Kedersha N: RNA granules. J Cell Biol 172: 803-808, 2006.

30. Huang C, Chen Y, Dai H, Zhang H, Xie M, Zhang H, Chen F, Kang X, Bai X and Chen Z: UBAP2L arginine methylation by PRMT1 modulates stress granule assembly. Cell Death Differ 27: 227-241, 2020.

31. Tsai WC, Gayatri S, Reineke LC, Sbardella G, Bedford MT and Lloyd RE: Arginine demethylation of G3BP1 promotes stress granule assembly. J Biol Chemistry 291: 22671-22685, 2016. 
32. Kedersha N and Anderson P: Stress granules: Sites of mRNA triage that regulate mRNA stability and translatability. Biochem Soc Trans 30: 963-969, 2002.

33. Mateju D, Eichenberger B, Voigt F, Eglinger J, Roth G and Chao JA: Single-molecule imaging reveals translation of mRNAs localized to stress granules. Cell 183: 1801-1812.e13, 2020.

34. Arimoto K, Fukuda H, Imajoh-Ohmi S, Saito H and Takekawa M: Formation of stress granules inhibits apoptosis by suppressing stress-responsive MAPK pathways. Nat Cell Biol 10: 1324-1332, 2008.

35. Park YJ, Choi DW, Cho SW, Han J, Yang S and Choi CY: Stress granule formation attenuates RACK1-mediated apoptotic cell death induced by morusin. Int J Mol Sci 21: 5360, 2020.

36. Panas MD, Ivanov P and Anderson P: Mechanistic insights into mammalian stress granule dynamics. J Cell Biol 215: 313-323, 2016.

37. Tourrière H, Chebli K, Zekri L, Courselaud B, Blanchard JM, Bertrand E and Tazi J: The RasGAP-associated endoribonuclease G3BP assembles stress granules. J Cell Biol 160: 823-831, 2003.

38. Omer A, Patel D, Moran JL, Lian XJ, Di Marco S and Gallouzi IE: Autophagy and heat-shock response impair stress granule assembly during cellular senescence. Mech Ageing Dev 192: 111382, 2020.

39. Omer A, Barrera MC, Moran JL, Lian XJ, Di Marco S, Beausejour C and Gallouzi IE: G3BP1 controls the senescence-associated secretome and its impact on cancer progression. Nat Commun 11: 4979, 2020.

40. Coppé JP, Desprez PY, Krtolica A and Campisi J: The senescence-associated secretory phenotype: The dark side of tumor suppression. Annu Rev Pathol 5: 99-118, 2010.

41. Anderson P, Kedersha N and Ivanov P: Stress granules, P-bodies and cancer. Biochim Biophys Acta 1849: 861-870, 2015.

42. El-Naggar AM and Sorensen PH: Translational control of aberrant stress responses as a hallmark of cancer. J Pathol 244 650-666, 2018

43. Vilas-Boas Fde A, da Silva AM, de Sousa LP, Lima KM, Vago JP, Bittencourt LF, Dantas AE, Gomes DA, Vilela MC, Teixeira MM and Barcelos LS: Impairment of stress granule assembly via inhibition of the eIF2alpha phosphorylation sensitizes glioma cells to chemotherapeutic agents. J Neurooncol 127: 253-260, 2016

44. Fournier MJ, Gareau C and Mazroui R: The chemotherapeutic agent bortezomib induces the formation of stress granules. Cancer Cell Int 10: 12, 2010.

45. Gao X, Jiang L, Gong Y, Chen X, Ying M, Zhu H, He Q, Yang B and Cao J: Stress granule: A promising target for cancer treatment. Br J Pharmacol 176: 4421-4433, 2019.

46. Khan FH, Dervan E, Bhattacharyya DD, McAuliffe JD, Miranda KM and Glynn SA: The role of nitric oxide in cancer: Master regulator or NOt? Int J Mol Sci 21: 9393, 2020.

47. Alam U and Kennedy D: G3BP1 and G3BP2 regulate translation of interferon-stimulated genes: IFITM1, IFITM2 and IFITM3 in the cancer cell line MCF7. Mol Cell Biochem 459: 189-204, 2019.

48. Gupta N, Badeaux M, Liu Y, Naxerova K, Sgroi D, Munn LL, Jain RK and Garkavtsev I: Stress granule-associated protein G3BP2 regulates breast tumor initiation. Proc Natl Acad Sci USA 114: 1033-1038, 2017.

49. Somasekharan SP, El-Naggar A, Leprivier G, Cheng H, Hajee S, Grunewald TG, Zhang F, Ng T, Delattre O, Evdokimova V, et al: YB-1 regulates stress granule formation and tumor progression by translationally activating G3BP1. J Cell Biol 208: 913-929, 2015.

50. Ramachandran B, Stabley JN, Cheng SL, Behrmann AS, Gay A, Li L, Mead M, Kozlitina J, Lemoff A, Mirzaei H, et al: A GTPase-activating protein-binding protein (G3BP1)/antiviral protein relay conveys arteriosclerotic Wnt signals in aortic smooth muscle cells. J Biol Chem 293: 7942-7968, 2018.

51. Schneider JW, Oommen S, Qureshi MY, Goetsch SC, Pease DR Sundsbak RS, Guo W, Sun M, Sun H, Kuroyanagi H, et al: Dysregulated ribonucleoprotein granules promote cardiomyopathy in RBM20 gene-edited pigs. Nat Med 26: 1788-1800, 2020

52. Smit M, Coetzee AR and Lochner A: The pathophysiology of myocardial ischemia and perioperative myocardial infarction. J Cardiothorac Vasc Anesth 34: 2501-2512, 2020.

53. Garikipati VNS, Verma SK, Cheng Z, Liang D, Truongcao MM, Cimini M, Yue Y, Huang G, Wang C, Benedict C, et al: Circular RNA CircFndc $3 b$ modulates cardiac repair after myocardial infarction via FUS/VEGF-A axis. Nat Commun 10: 4317, 2019.

54. Mahboubi H and Stochaj U: Cytoplasmic stress granules: Dynamic modulators of cell signaling and disease. Biochim Biophys Acta Mol Basis Dis 1863: 884-895, 2017.
55. Yoneyama M, Jogi $\mathrm{M}$ and Onomoto $\mathrm{K}$ : Regulation of antiviral innate immune signaling by stress-induced RNA granules. J Biochem 159: 279-286, 2016

56. Xu S, Chen D, Chen D, Hu Q, Zhou L, Ge X, Han J, Guo X and Yang H: Pseudorabies virus infection inhibits stress granules formation via dephosphorylating eIF2 $\alpha$. Vet Microbiol 247: 108786, 2020.

57. Khong A, Kerr CH, Yeung CHL, Keatings K, Nayak A, Allan DW and Jan E: Disruption of stress granule formation by the multifunctional cricket paralysis virus 1A protein. J Virol 91: e01779-16, 2017

58. Visser LJ, Medina GN, Rabouw HH, de Groot RJ, Langereis MA, de Los Santos T and van Kuppeveld FJM: Foot-and-Mouth disease virus leader protease cleaves G3BP1 and G3BP2 and inhibits stress granule formation. J Virol 93: e00922-18, 2019.

59. Dougherty JD, Tsai WC and Lloyd RE: Multiple poliovirus proteins repress cytoplasmic RNA granules. Viruses 7: 6127-6140, 2015

60. Yang X, Hu Z, Fan S, Zhang Q, Zhong Y, Guo D, Qin Y and Chen M: Picornavirus $2 \mathrm{~A}$ protease regulates stress granule formation to facilitate viral translation. PLoS Pathog 14: e1006901, 2018.

61. Le Sage V, Cinti A, McCarthy S, Amorim R, Rao S, Daino GL Tramontano E, Branch DR and Mouland AJ: Ebola virus VP35 blocks stress granule assembly. Virology 502: 73-83, 2017.

62. Savastano A, Ibáñez de Opakua A, Rankovic M and Zweckstetter M: Nucleocapsid protein of SARS-CoV-2 phase separates into RNA-rich polymerase-containing condensates. Nat Commun 11: 6041, 2020.

63. Wang J, Shi C, Xu Q and Yin H: SARS-CoV-2 nucleocapsid protein undergoes liquid-liquid phase separation into stress granules through its N-terminal intrinsically disordered region. Cell Discov 7: 5, 2021.

64. Lu S, Ye Q, Singh D, Cao Y, Diedrich JK, Yates JR III, Villa E, Cleveland DW and Corbett KD: The SARS-CoV-2 nucleocapsid phosphoprotein forms mutually exclusive condensates with RNA and the membrane-associated M protein. Nat Commun 12: 502 , 2021.

65. Prasad K, Alasmari AF, Ali N, Khan R, Alghamdi A and Kumar V: Insights into the SARS-CoV-2-Mediated alteration in the stress granule protein regulatory networks in humans. Pathogens 10: 1459, 2021.

66. Thoms M, Buschauer R, Ameismeier M, Koepke L, Denk T, Hirschenberger M, Kratzat H, Hayn M, Mackens-Kiani T, Cheng J, et al: Structural basis for translational shutdown and immune evasion by the Nsp1 protein of SARS-CoV-2. Science 369: 1249-1255, 2020.

67. Shi M, Wang L, Fontana P, Vora S, Zhang Y, Fu TM, Lieberman J and $\mathrm{Wu} \mathrm{H}$ : SARS-CoV-2 Nsp1 suppresses host but not viral translation through a bipartite mechanism. bioRxiv 2020: 302901, 2020.

68. Schubert K, Karousis ED, Jomaa A, Scaiola A, Echeverria B, Gurzeler LA, Leibundgut M, Thiel V, Mühlemann O and Ban N: SARS-CoV-2 Nspl binds the ribosomal mRNA channel to inhibit translation. Nat Struct Mol Biol 27: 959-966, 2020.

69. Nakagawa K, Narayanan K, Wada M and Makino S: Inhibition of stress granule formation by middle east respiratory syndrome coronavirus $4 \mathrm{a}$ accessory protein facilitates viral translation, leading to efficient virus replication. J Virol 92: e00902-18, 2018.

70. Callaway E: Heavily mutated omicron variant puts scientists on alert. Nature 600: 21, 2021.

71. Dudman J and Qi X: Stress granule dysregulation in amyotrophic lateral sclerosis. Front Cell Neurosci 14: 598517, 2020.

72. Anderson EN, Gochenaur L, Singh A, Grant R, Patel K, Watkins S, Wu JY and Pandey UB: Traumatic injury induces stress granule formation and enhances motor dysfunctions in ALS/FTD models. Hum Mol Genet 27: 1366-1381, 2018.

73. Ayuso MI, Martínez-Alonso E, Regidor I and Alcázar A: Stress granule induction after brain ischemia is independent of eukaryotic translation initiation factor (eIF) $2 \alpha$ phosphorylation and is correlated with a decrease in eIF4B and eIF4E proteins. J Biol Chemistry 291: 27252-27264, 2016.

74. Correia AS, Patel P, Dutta K and Julien JP: Inflammation induces TDP-43 mislocalization and aggregation. PLoS One 10: $\mathrm{e} 0140248,2015$

75. Cao X, Jin X and Liu B: The involvement of stress granules in aging and aging-associated diseases. Aging Cell 19: e13136, 2020 .

76. Cruz A, Verma M and Wolozin B: The Pathophysiology of tau and stress granules in disease. Adv Exp Med Biol 1184: 359-372, 2019. 
77. Webber CJ, Lei SE and Wolozin B: The pathophysiology of neurodegenerative disease: Disturbing the balance between phase separation and irreversible aggregation. Prog Mol Biol Transl Sci 174: 187-223, 2020.

78. Rozpędek-Kamińska W, Siwecka N, Wawrzynkiewicz A, Wojtczak R, Pytel D, Diehl JA and Majsterek I: The PERK-dependent molecular mechanisms as a novel therapeutic target for neurodegenerative diseases. Int J Mol Sci 21: 2108, 2020.

79. Ma T, Trinh MA, Wexler AJ, Bourbon C, Gatti E, Pierre P, Cavener DR and Klann E: Suppression of eIF2 $\alpha$ kinases alleviates Alzheimer's disease-related plasticity and memory deficits. Nat Neurosci 16: 1299-1305, 2013.

80. Kim HJ, Raphael AR, LaDow ES, McGurk L, Weber RA, Trojanowski JQ, Lee VM, Finkbeiner S, Gitler AD and Bonini NM: Therapeutic modulation of eIF2 $\alpha$ phosphorylation rescues TDP-43 toxicity in amyotrophic lateral sclerosis disease models. Nat Genet 46: 152-160, 2014.

81. Banani SF, Lee HO, Hyman AA and Rosen MK: Biomolecular condensates: Organizers of cellular biochemistry. Nat Rev Mol Cell Biol 18: 285-298, 2017

82. Flores BN, Li X, Malik AM, Martinez J, Beg AA and Barmada SJ: An intramolecular salt bridge linking TDP43 RNA binding, protein stability, and TDP43-dependent neurodegeneration. Cell Rep 27: 1133-1150.e8, 2019.

83. Archbold HC, Jackson KL, Arora A, Weskamp K, Tank EM, Li X, Miguez R, Dayton RD, Tamir S, Klein RL and Barmada SJ: TDP43 nuclear export and neurodegeneration in models of amyotrophic lateral sclerosis and frontotemporal dementia. Sci Rep 8: 4606, 2018

84. Suk TR and Rousseaux MWC: The role of TDP-43 mislocalization in amyotrophic lateral sclerosis. Mol Neurodegener 15: 45, 2020.

85. Tomé SO, Vandenberghe R, Ospitalieri S, Van Schoor E, Tousseyn T, Otto M, von Arnim CAF and Thal DR: Distinct molecular patterns of TDP-43 pathology in Alzheimer's disease: Relationship with clinical phenotypes. Acta Neuropathol Commun 8: 61, 2020.

86. Vanderweyde T, Apicco DJ, Youmans-Kidder K, Ash PEA, Cook C, Lummertz da Rocha E, Jansen-West K, Frame AA, Citro A, Leszyk JD, et al: Interaction of tau with the RNA-binding Protein TIA1 regulates tau pathophysiology and toxicity. Cell Rep 15: 1455-1466, 2016

87. Apicco DJ, Ash PEA, Maziuk B, LeBlang C, Medalla M, Al Abdullatif A, Ferragud A, Botelho E, Ballance HI, Dhawan U, et al: Reducing the RNA binding protein TIA1 protects against tau-mediated neurodegeneration in vivo. Nat Neurosci 21: 72-80, 2018.

88. Gal J, Kuang L, Barnett KR, Zhu BZ, Shissler SC, Korotkov KV, Hayward LJ, Kasarskis EJ and Zhu H: ALS mutant SOD1 interacts with G3BP1 and affects stress granule dynamics. Acta Neuropathol 132: 563-576, 2016.

89. Sidibé H, Dubinski A and Vande Velde C: The multi-functional RNA-binding protein G3BP1 and its potential implication in neurodegenerative disease. J Neurochem 157: 944-962, 2021.

90. Gogia N, Sarkar A, Mehta AS, Ramesh N, Deshpande P, Kango-Singh M, Pandey UB and Singh A: Inactivation of Hippo and cJun-N-terminal Kinase (JNK) signaling mitigate FUS mediated neurodegeneration in vivo. Neurobio Dis 140: 104837, 2020.

91. Picchiarelli G, Demestre M, Zuko A, Been M, Higelin J, Dieterlé S, Goy MA, Mallik M, Sellier C, Scekic-Zahirovic J, et al: FUS-mediated regulation of acetylcholine receptor transcription at neuromuscular junctions is compromised in amyotrophic lateral sclerosis. Nat Neurosci 22: 1793-1805, 2019.

92. Fifita JA, Zhang KY, Galper J, Williams KL, McCann EP, Hogan AL, Saunders N, Bauer D, Tarr IS, Pamphlett R, et al Genetic and pathological assessment of hnRNPA1, hnRNPA2/B1, and hnRNPA3 in familial and sporadic amyotrophic lateral sclerosis. Neurodegener Dis 17: 304-312, 2017.
93. Kim HJ, Kim NC, Wang YD, Scarborough EA, Moore J, Diaz Z, MacLea KS, Freibaum B, Li S, Molliex A, et al: Mutations in prion-like domains in hnRNPA2B1 and hnRNPA1 cause multisystem proteinopathy and ALS. Nature 495: 467-473, 2013.

94. Montalbano M, McAllen S, Cascio FL, Sengupta U, Garcia S, Bhatt N, Ellsworth A, Heidelman EA, Johnson OD, Doskocil S and Kayed R: TDP-43 and tau oligomers in Alzheimer's disease, amyotrophic lateral sclerosis, and frontotemporal dementia. Neurobiol Dis 146: 105130, 2020.

95. Zhao M, Kim JR, van Bruggen R and Park J: RNA-binding proteins in amyotrophic lateral sclerosis. Mol Cells 41: 818-829, 2018.

96. Mackenzie IR, Nicholson AM, Sarkar M, Messing J, Purice MD, Pottier C, Annu K, Baker M, Perkerson RB, Kurti A, et al: TIA1 mutations in amyotrophic lateral sclerosis and frontotemporal dementia promote phase separation and alter stress granule dynamics. Neuron 95: 808-816.e9, 2017.

97. Chew J, Gendron TF, Prudencio M, Sasaguri H, Zhang YJ, Castanedes-Casey M, Lee CW, Jansen-West K, Kurti A, Murray ME, et al: Neurodegeneration. C9ORF72 repeat expansions in mice cause TDP-43 pathology, neuronal loss, and behavioral deficits. Science 348: 1151-1154, 2015.

98. Chew J, Cook C, Gendron TF, Jansen-West K, Del Rosso G, Daughrity LM, Castanedes-Casey M, Kurti A, Stankowski JN, Disney MD, et al: Aberrant deposition of stress granule-resident proteins linked to C9orf72-associated TDP-43 proteinopathy. Mol Neurodegener 14: 9, 2019.

99. Chitiprolu M, Jagow C, Tremblay V, Bondy-Chorney E, Paris G, Savard A, Palidwor G, Barry FA, Zinman L, Keith J, et al: A complex of C9ORF72 and p62 uses arginine methylation to eliminate stress granules by autophagy. Nat Commun 9: 2794 , 2018.

100. Deng Z, Lim J, Wang Q, Purtell K, Wu S, Palomo GM, Tan H, Manfredi G, Zhao Y, Peng J, et al: ALS-FTLD-linked mutations of SQSTM1/p62 disrupt selective autophagy and NFE2L2/NRF2 anti-oxidative stress pathway. Autophagy 16: 917-931, 2020.

101. Jiang Z, Belforte JE, Lu Y, Yabe Y, Pickel J, Smith CB, Je HS, Lu B and Nakazawa K: eIF2alpha Phosphorylation-dependent translation in CA1 pyramidal cells impairs hippocampal memory consolidation without affecting general translation. J Neurosci 30: 2582-2594, 2010.

102. Trinh MA, Ma T, Kaphzan H, Bhattacharya A, Antion MD, Cavener DR, Hoeffer CA and Klann E: The eIF2 $\alpha$ kinase PERK limits the expression of hippocampal metabotropic glutamate receptor-dependent long-term depression. Learn Mem 21: 298-304, 2014

103. Hijioka M, Inden M, Yanagisawa D and Kitamura Y: DJ-1/PARK7: A new therapeutic target for neurodegenerative disorders. Biol Pharm Bull 40: 548-552, 2017.

104. Repici M, Hassanjani M, Maddison DC, Garção P, Cimini S, Patel B, Szegö ÉM, Straatman KR, Lilley KS, Borsello T, et al: The Parkinson's disease-linked protein DJ-1 associates with cytoplasmic mRNP granules during stress and neurodegeneration. Mol Neurobiol 56: 61-77, 2019.

105. Ma J, Wu R, Zhang Q, Wu JB, Lou J, Zheng Z, Ding JQ and Yuan Z: DJ-1 interacts with RACK1 and protects neurons from oxidative-stress-induced apoptosis. Biochem J 462: 489-497, 2014. International (CC BY-NC-ND 4.0) License. 